\title{
COMORBIDITIES IN DRIVERS WITH PARKINSON DISEASE
}

\author{
Maud Ranchet ${ }^{1}$, Mark Tant ${ }^{2}$, Abiodun E. Akinwuntan ${ }^{1}$, Hannes Devos ${ }^{1}$ \\ ${ }^{1}$ Georgia Regents University, Augusta, Georgia, USA \\ ${ }^{2}$ Belgian Road Safety Institute, Brussels, Belgium \\ Email: mranchet@gru.edu
}

\begin{abstract}
Summary: Previous studies have shown that comorbidities have an impact on driving performance in older adults. No study has established the relationships between comorbidities and driving in persons with Parkinson disease (PD). The aims of this study were (1) to report the types of comorbidity in a group of 111 drivers with PD and (2) to identify whether the comorbidity associated with PD is a predictor of overall fitness-to-drive decisions, crashes, and validity duration of driving license. Results showed that 72 participants (64.9\%) had only Parkinson disease, and 39 (35.1\%) participants had one or more medical conditions in addition to PD. The most frequent comorbidities were visual disorders $(26.4 \%)$, heart and blood disorders (16.2\%), neurological disorders other than PD $(11.8 \%)$, and locomotor disorders $(11.8 \%)$. Contrarily to what we expected, we did not find any significant associations between comorbidities and overall fitness-to-drive decisions, car crashes, or validity duration of driving license. We conclude that in this sample of drivers with PD, comorbidity was not a significant predictor of overall fitness-to-drive decisions.
\end{abstract}

\section{INTRODUCTION}

Mobility is an important activity of daily living that affects physical, emotional, and social wellbeing (James et al., 2011). In broad terms, mobility is the purposeful movement through the environment and spans a continuum from total immobility (e.g., bed-ridden) to travels to distant locations (Ross et al., 2013).

Comorbidity, which is defined as the presence of one or more additional disorders co-occurring with a primary disease (Valderas et al., 2009), can have serious implications on mobility. A variety of comorbid conditions, such as eye disease, heart disease, and fatigue are related to mobility limitations in older adults (Popescu et al., 2011; Yeom et al., 2008). These comorbid conditions may impact gait, balance, but also other modes of mobility such as driving (Papa et al., 2014; Popescu et al., 2011). The age-related changes in brain, musculoskeletal, visual, and auditory functions may be exacerbated when occurring concurrently with Parkinson disease (PD) (King et al., 2014; Sagna et al., 2014). PD is a progressive neurological condition characterized by motor and non-motor symptoms which may adversely affect driving performance (Devos et al., 2013; Ranchet et al., 2013; Uc et al., 2009).

A recent study reported a high frequency of comorbidities in people with mild to moderate PD (King et al., 2014). King et al. (2014) found eyes and ears (89\%), psychiatric (68\%), musculoskeletal (64\%), lower gastrointestinal (62\%), respiratory $(60.5 \%)$, upper gastrointestinal $(59.2 \%)$ and genitourinary $(53.9 \%)$ conditions to be most frequently reported in association with PD. Visser et al. (2004) reported that $84 \%$ of patients with PD had one or more comorbidities 
such as lower gastrointestinal (55\%), genitourinary (52\%), musculoskeletal (48\%) and eyes, ears, throat, and larynx (48\%) diseases. The frequency of comorbidity was significantly related to measures of mobility including gait, balance, and physical function (King et al., 2014). To date, the relationship between comorbidities and fitness-to-drive decisions, car crashes, and other driving measures remains unclear.

The aims of this study were (1) to report the types of comorbidities in a group of drivers with PD and (2) to identify whether the comorbidities associated with PD are related to overall fitness-todrive decisions, car crashes, and validity duration of driving license.

\section{METHODS}

\section{Population}

Recruitment and eligibility. The data of all clients who were evaluated at the Center of Evaluation of Fitness to drive and Car Adaptations (CARA) of the Belgian Road Safety Institute in the period January to December 2013 were reviewed for eligibility. Clients were included if (1) they were diagnosed with Parkinson's disease; (2) had a valid driver's license; (3) and met the minimum visual acuity requirements of fitness to drive in Belgium (20/40 binocular acuity). The comorbidity was recorded by the referring physician and categorized into 12 groups: (1) neurological condition other than PD (e.g., dementia, stroke...); (2) locomotor disorders; (3) visual dysfunction; (4) heart and blood disorders; (5) mental disorders; (6) epilepsy; (7) diabetes; (8) psychotropic substance abuse; (9) vestibular system disorders; (10) implants; (11) renal and hepatic impairment; and (12) pathological somnolence. This study was approved by the Georgia Regents University Institutional Review Board (IRB-A).

\section{Outcome variables}

Overall fitness-to-drive decision. The overall fitness-to-drive evaluation performed at CARA included a review of the medical history, an assessment of visual functions and on-road driving skills, and if needed, a neuropsychological evaluation. After the CARA evaluation, participants were categorized as pass when they were "fit to drive without restrictions" or "fit to drive with restrictions" (i.e. restricted use in time, distance or speed) and categorized as fail when they were "unfit to drive". Fifty-four patients $(48.6 \%)$ came to CARA for an extension of the drivers' license validity period, $50(45.0 \%)$ because of change in medical situation, $2(1.8 \%)$ were referred by their insurance company or a medical expert) and $5(4.5 \%)$ for unknown reasons. Car crashes. Participants were asked if they were involved in a car crash in the five years prior to the CARA evaluation (dichotomous variable: "yes" or "no").

Validity duration of driver's license. This variable was calculated as the difference between the allocated expiration date of the driving license and date of assessment.

\section{Data analysis}

To investigate the effect of comorbidities on overall fitness-to-drive decisions, car crashes, and the validity duration of the driver's license in PD, participants were divided in 2 groups: (1) people with PD only and (2) people with PD and one or more medical conditions. Univariate 
Analysis of Variance (ANOVA) was used to compare age between the 2 groups. $\mathrm{Chi}^{2}$ was used for categorical variables to determine associations between comorbidities and overall fitness-todrive decisions or car crashes. Spearman correlations were performed between the number of comorbidities and the validity duration of driver's license. Binomial logistic regression or linear regression analyses were used to investigate whether comorbidities are predictors of overall fitness-to-drive decisions, car crashes, and validity duration of driver's license. P values $\leq 0.05$ were applied to all our statistical analyses. All analyses were performed with SPSS 21.0 statistical software.

\section{RESULTS}

\section{Population}

One hundred eleven (111) clients were PD (91 men/ 20 women) were evaluated by CARA. The mean age was $68.05 \pm 10.92$ years, with a range from 35 to 96 years. Among 98 participants, 64 $(65.3 \%)$ were declared "fit to drive without restrictions", 20 were $(20.4 \%)$ "fit to drive with restrictions" and $14(14.3 \%)$ were "unfit to drive" (missing variables: $n=13)$. Eighteen patients $(16.2 \%)$ reported having one or more crashes in the 5 years prior to testing and $76(68.4 \%)$ reported no crashes. Seventeen (15.3\%) did not report information with regard to car crashes.

\section{Frequency and types of comorbidity in drivers with PD}

Seventy-two people (64.9\%) had only PD and 39 (35.1\%) people had 1 or more comorbidities in addition to PD. An increased number of comorbidities was associated with a reduced number of people $\left(\mathrm{Chi}^{2}=49.7, \mathrm{p}<0.001\right)$. No significant differences in terms of age between the 2 groups were found (PD group: 67.1 \pm 11.1 years; PD group +1 or more medical condition: $69.8 \pm 10.5$, $\mathrm{F}=1.5, \mathrm{p}=0.2)$. Figure 1 shows the percentage of drivers with one medical condition in addition to $\mathrm{PD}$.

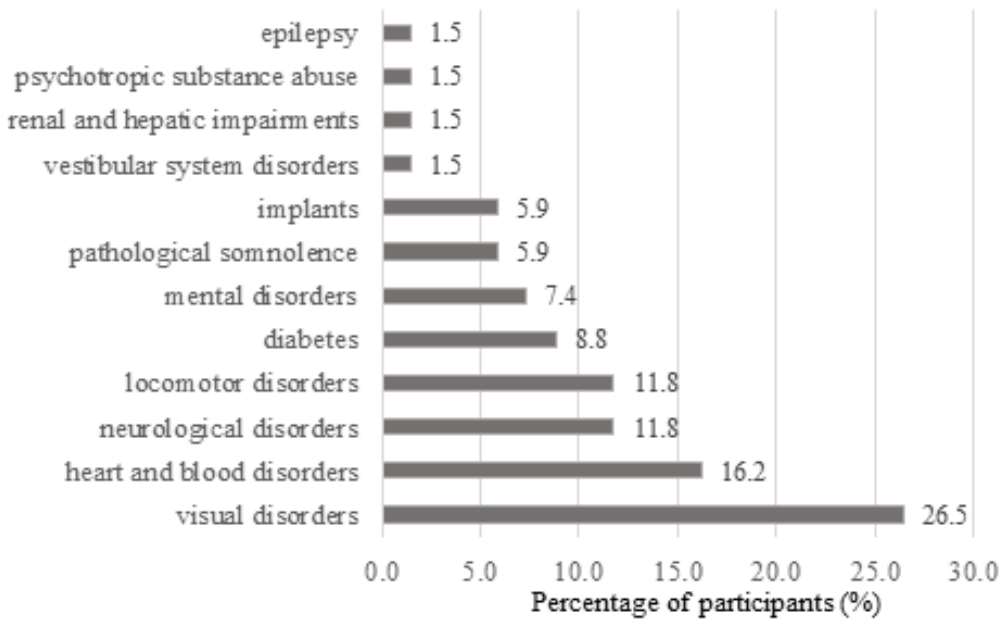

Figure 1. Percentage of drivers with another type of medical condition than PD 
The four most frequent medical conditions reported in this sample were visual disorders $(\mathrm{n}=18$, $26.5 \%)$, heart and blood disorders $(\mathrm{n}=11,16.2 \%)$, neurological disorders other than $\mathrm{PD}(\mathrm{n}=8$, $11.8 \%)$, and locomotor disorders $(n=8,11.8 \%)$, followed by diabetes $(n=6,8.8 \%)$, mental disorders $(n=5,7.4 \%)$, pathological somnolence $(n=4,5.9 \%)$, implants $(n=4,5.8 \%)$, vestibular system disorders $(n=1,1.5 \%)$, renal and hepatic impairments $(n=1,1.5 \%)$, psychotropic substance abuse $(n=1,1.5 \%)$, and epilepsy $(n=1,1.5 \%)$.

\section{Relationships between comorbidities and fitness-to-drive decisions, car crashes, and validity duration of the driving license in PD patients}

No significant associations were found between comorbidities and fitness-to-drive decisions $\left(\mathrm{Chi}^{2}=0.03, \mathrm{p}=0.86\right)$ and car crashes $\left(\mathrm{Chi}^{2}=0.50, \mathrm{p}=0.48\right)$ (Table 1 and Table 2 , respectively).

Table 1. Association between comorbidities and overall fitness-to-drive decisions

\begin{tabular}{|cccc|}
\hline & \multicolumn{3}{c|}{ Overall fitness-to-drive decisions } \\
\cline { 2 - 4 } & Pass & Fail & $\mathrm{p}$ \\
\hline only PD & $56(86.2 \%)$ & $9(13.8 \%)$ & 0.86 \\
1 or more additional medical conditions & $28(84.8 \%)$ & $5(15.2 \%)$ & \\
\hline
\end{tabular}

Table 2. Association between comorbidities and car crashes

\begin{tabular}{cccc}
\hline & \multicolumn{2}{c}{ Car crashes } & \\
\cline { 2 - 4 } & No & Yes & $\mathrm{p}$ \\
\hline only PD & $49(83.1 \%)$ & $10(16.9 \%)$ & 0.48 \\
\hline 1 or more additional medical conditions & $27(71.4 \%)$ & $8(22.9 \%)$ & \\
\hline
\end{tabular}

Similarly, comorbidities did not predict overall fitness-to-drive decisions $(\mathrm{B}=0.11$, Odds Ratio $(\mathrm{OR})=1.11, \mathrm{CI} 95 \%$ : $0.34-3.63, \mathrm{p}=0.86)$ and car crashes $(\mathrm{B}=0.37, \mathrm{OR}=1.452, \mathrm{CI} 95 \%: 0.51$ $-4.11, \mathrm{p}=0.48)$ in the logistic regression analysis $(\mathrm{n}=98)$.

No significant correlation was found between the number of comorbidities and driving license duration ( $r$ ho $=-0.02, p=0.87$ ). Furthermore, comorbidities did not predict the validity duration of driver's license in the linear regression model $(\mathrm{B}=-36.3$, CI 95\%: $-210.56-137.92, \mathrm{p}=0.68)$.

\section{DISCUSSION}

The main objective of this study was to identify whether comorbidities are related to overall fitness-to-drive decisions, self-reported car crashes and validity duration of the driver's license in a sample of 111 PD people evaluated by CARA in 2013. The interest of using a populationbased approach was to include a heterogeneous group of drivers with PD who have one or more comorbidities, which are often considered an exclusion criterion in most studies on driving and PD (Classen et al., 2014; Devos et al., 2007; Ranchet et al., 2013; Uc et al., 2009). Consequently, it is often difficult to generalize the findings to the population of drivers with PD who often experience age-related comorbidities. Several studies have shown comorbidities as predictor of 
driving performance, driving pattern and crash involvement in older adults (Papa et al., 2014) or in individuals with neurological conditions (Devos et al., 2010). For instance, Devos et al. (2010) showed that patients who gave up driving in the five years after their stroke had more comorbidity than those who were still driving. However, no study has specifically explored the impact of comorbidities on driving measures in a group of active drivers with PD.

The most frequent medical conditions in our sample were visual disorders $(26.5 \%)$, heart and blood disorders (16.2\%), neurological disorders other than PD (11.8\%) and locomotor disorders $(11.8 \%)$, which is in accordance with the type of comorbidity reported in the literature (King et al., 2014; Visser et al., 2004). Deficit in visual functions and heart and blood disorders are common among elderly people and may be exacerbated by PD. Yet, compared with previous studies (King et al., 2014; Visser et al., 2004), the proportion of people with PD having comorbidities in this sample (35.1\%) was relatively low. The participants in this study are still active drivers and may have presented with a better clinical picture than those in previous studies (King et al., 2014; Visser et al., 2004). Another reason may be that physicians only reported comorbidity that was expected to impact safe driving. Comorbidities related to the gastrointestinal system, genitourinary system, and throat and larynx diseases, which are frequently observed in PD (King et al., 2014), may have been underreported in our sample of drivers with PD.

Contrary to what we expected, we did not find any significant associations between comorbidities and overall fitness-to-drive decisions. The lack of significant results might be due to the binary measure of driving safety (pass/fail). Similarly, no significant associations were found between comorbidities and self-reported car crashes, and validity duration of the driver's license, which may be due to the small number of PD drivers having one or more comorbidities. As previously said, this number is relatively low compared to other studies in PD people (King et al., 2014; Visser et al., 2004). Another reason might be that comorbidities associated with our PD group were not severe enough to have an impact on the driving outcomes measures in our study. In the study of King et al. (2014), 60\% of PD people reported mild complaints of eyes and ears whereas $43 \%$ reported severe psychiatric comorbidity. The severity of the comorbidities was significantly associated with mobility measures. Further studies with larger sample sizes that take into account severity indices of comorbidity are therefore warranted.

\section{Conclusions}

Most studies on driving and PD have used stringent eligibility criteria, which make it difficult to generalize their findings to the population of drivers with PD. The current study used a cohort of community-dwelling drivers with PD to explore the associations between comorbidity and driving. In this sample, we did not find that comorbidity was a significant predictor of overall fitness-to-drive decisions, car crashes and validity duration of the driver's license. Further investigation will explore the impact of comorbidities on driving safety in PD.

\section{AKNOWLEDGEMENTS}

The authors thank the CARA staff for the fitness to drive evaluations. 


\section{REFERENCES}

Classen, S., Brumback, B., Monahan, M., Malaty, I. I., Rodriguez, R. L., Okun, M. S., \& McFarland, N. R. (2014). Driving errors in Parkinson's disease: moving closer to predicting on-road outcomes. The American Journal Of Occupational Therapy: Official Publication Of The American Occupational Therapy Association, 68(1), 77-85. doi: 10.5014/ajot.2014.008698

Devos, H., Akinwuntan, A. E., Nieuwboer, A., Ringoot, I., Van Berghen, K., Tant, M., Kiekens, C., \& De Weerdt, W. (2010). Effect of simulator training on fitness-to-drive after stroke: a 5year follow-up of a randomized controlled trial. Neurorehabilitation Neural Repair, 24(9), 843-850. doi: 10.1177/1545968310368687

Devos, H., Vandenberghe, W., Nieuwboer, A., Tant, M., Baten, G., \& De Weerdt, W. (2007). Predictors of fitness to drive in people with Parkinson disease. Neurology, 69(14), 14341441. doi: 69/14/1434 [pii]10.1212/01.wnl.0000277640.58685.fc

Devos, H., Vandenberghe, W., Tant, M., Akinwuntan, A. E., De Weerdt, W., Nieuwboer, A., \& Uc, E. Y. (2013). Driving and off-road impairments underlying failure on road testing in Parkinson's disease. Movement Disorders, 28(14), 1949-1956. doi: 10.1002/mds.25701

James, B. D., Boyle, P. A., Buchman, A. S., \& Bennett, D. A. (2011). Relation of late-life social activity with incident disability among community-dwelling older adults. Journal of Gerontology: Series A, Biological Sciences Medical Sciences, 66(4), 467-473. doi: 10.1093/gerona/glq231

King, L. A., Priest, K. C., Nutt, J., Chen, Y., Chen, Z., Melnick, M., \& Horak, F. (2014). Comorbidity and Functional Mobility in Persons with Parkinson Disease. Archive of Physical Medicine and Rehabilitation. doi: 10.1016/j.apmr.2014.07.396

Papa, M., Boccardi, V., Prestano, R., Angellotti, E., Desiderio, M., Marano, L., Rizzo, M. R., \& Paolisso, G. (2014). Comorbidities and crash involvement among younger and older drivers. PLoS One, 9(4), e94564. doi: 10.1371/journal.pone.0094564

Popescu, M. L., Boisjoly, H., Schmaltz, H., Kergoat, M. J., Rousseau, J., Moghadaszadeh, S., Djafari, F., \& Freeman, E. E. (2011). Age-related eye disease and mobility limitations in older adults. Investigative Ophthalmoly and Visual Science, 52(10), 7168-7174. doi: 10.1167/iovs.11-7564

Ranchet, M., Paire-Ficout, L., Uc, E. Y., Bonnard, A., Sornette, D., \& Broussolle, E. (2013). Impact of specific executive functions on driving performance in people with Parkinson's disease. Movement Disorders, 28(14), 1941-1948. doi: 10.1002/mds.25660

Ross, L. A., Schmidt, E. L., \& Ball, K. (2013). Interventions to maintain mobility: What works? Accident Analysis and Prevention, 61, 167-196. doi: 10.1016/j.aap.2012.09.027

Sagna, A., Gallo, J. J., \& Pontone, G. M. (2014). Systematic review of factors associated with depression and anxiety disorders among older adults with Parkinson's disease. Parkinsonism and Related Disorders, 20(7), 708-715. doi: 10.1016/j.parkreldis.2014.03.020 
Uc, E. Y., Rizzo, M., Johnson, A. M., Dastrup, E., Anderson, S. W., \& Dawson, J. D. (2009). Road safety in drivers with Parkinson disease. Neurology, 73, 2112-2119. doi: 10.1212/WNL.0b013e3181c67b77

Valderas, J. M., Starfield, B., Sibbald, B., Salisbury, C., \& Roland, M. (2009). Defining comorbidity: implications for understanding health and health services. The Annals of Family Medicine, 7(4), 357-363. doi: 10.1370/afm.983

Visser, M., Marinus, J., van Hilten, J. J., Schipper, R. G., \& Stiggelbout, A. M. (2004). Assessing comorbidity in patients with Parkinson's disease. Movement Disorders, 19(7), 824-828. doi: $10.1002 / \mathrm{mds} .20060$

Yeom, H. A., Fleury, J., \& Keller, C. (2008). Risk Factors for Mobility Limitation in Community-Dwelling Older Adults: A Social Ecological Perspective. Geriatric Nursing, 29(2), 133-140. doi: http://dx.doi.org/10.1016/j.gerinurse.2007.07.002 